\title{
Resultados a corto y largo plazo con la técnica de Karydakis para la enfermedad pilonidal sacrocoxígea*
}

\author{
Drs. ERNESTO MELKONIAN T.1,2, ALEJANDRO READI V. ${ }^{2}$, PATRICIO OPAZO S. ${ }^{2}$, \\ MARCELO RODRÍGUEZ G. ${ }^{2}$, DAVID CONTRERAS F. ${ }^{2}$, RODRIGO CÁPONA P. ${ }^{2}$, \\ ROLANDO REBOLLEDO A. ${ }^{2}$, CLAUDIO HEINE T. ${ }^{2}$ \\ Clínica Alemana. \\ 2 Unidad de Coloproctología, Servicio de Cirugía Hospital del Salvador. Facultad de Medicina, Universidad de Chile, \\ Campus Oriente. \\ Santiago, Chile.
}

\begin{abstract}
Short-and long-term results with Karydaki's technique for sacrococcygeal pilonidal disease
\end{abstract}

Introduction: Sacrococcygeal pilonidal cyst or sinus is a common condition in young people. There is still discussion about which of the many existing techniques is best suited to resolve this condition. Objective: To analyze the short-and long-term use of Karydaki's technique in our Hospital. Material and Methods: We analyzed prospective protocols from patients with pilonidal cyst with Karydaki's technique from June 2005 to August 2010. Clinical and telephonic follow-up was done to all patients and a satisfaction survey done to a random sample of the series. Results: We analyzed 62 patients with mean age of 24.5 years (15-45), being 33 men (53\%). Cysts were previously relapsed in $20.9 \%$ of cases. The average hospital stay was 2.5 days (1-3). Early complications occurred in $14.3 \%$ of patients being the most common seroma in $8 \%$ and dehiscence in $6.4 \%$. At a mean follow up of 38 months (12-62), relapse occurred in two patients (3.2\%), one at 6 months and the other at 3 years, being resolved one by a new Karydaki's technique and the other with marsupialization. Among the group surveyed for satisfaction, $75 \%$ found the technique satisfactory or very satisfactory in terms of aesthetics and $95 \%$ would recommend this technique. Conclusions: Karydaki's operation achieves with a simple and aesthetic technic a low recurrence and morbidity and a complete recovery after 15 days in most patients.

Key words: Sacrococcygeal cyst, pilonial sinus, surgical treatment, Karydakis technique.

\section{Resumen}

Introducción: El quiste o seno pilonidal sacrocoxígeo es una patología frecuente en gente joven. Aún hay discusión sobre cual de las múltiples técnicas existentes es la más adecuada para resolver esta patología. Objetivo: Analizar los resultados a corto y largo plazo con la utilización de la técnica de Karydakis en nuestro medio. Material y Método: Se analizan los protocolos prospectivos de pacientes intervenidos

*Recibido el 13 de junio de 2012 y aceptado para publicación el 25 de octubre de 2012.

Los autores no refieren conflictos de interés.

Correspondencia: Dr. Ernesto Melkonian T.

Los Lomajes 1131, Santiago, Chile.

melkonianherve@entelchile.net 
por quiste pilonidal con la técnica de Karydakis desde junio de 2005 hasta agosto de 2010. Se realizó seguimiento clínico y telefónico de los pacientes y una encuesta de satisfacción a una muestra aleatoria de la serie. Resultados: Se analizan 62 pacientes con promedio de edad de 24,5 años (15-45), siendo de ellos 33 hombres (53\%). En un 20,9\% de los casos eran quistes recidivados. La hospitalización promedio fue de 2,5 días (1-3). Un 14,3\% presentó complicaciones precoces siendo las más frecuentes el seroma (8\%) y dehiscencia de sutura $(6,4 \%)$. El seguimiento promedio fue de 38 meses (i:12-62). Dos pacientes tuvieron recidivas (3,2\%), a los 6 meses y a los 3 años, resolviéndose una con nueva técnica de Karydakis y la otra con marsupialización. Del grupo encuestado, el $75 \%$ consideró que la técnica fue satisfactoria o muy satisfactoria (estética) y el 95\% de los pacientes la recomendaría. Conclusiones: La operación de Karydakis logra con una técnica simple y estética una baja recidiva y morbilidad y una recuperación definitiva a los 15 días en la mayoría de los pacientes.

Palabras clave: Quiste sacrococcígeo, seno pilonidal, tratamiento quirúrgico, técnica de Karydakis.

\section{Introducción}

El quiste o seno pilonidal sacrocoxígeo es una patología frecuente en gente joven, desde la adolescencia hasta la cuarta década de la vida, produciendo molestias de importancia y ausentismo, derivadas de su infección y tradicionalmente de la alta recidiva post-operatoria. Estos hechos han motivado la descripción de múltiples técnicas, algunas más conservadoras desde cuidados de la zona sacrocoxígea hasta otras más definitivas, consistiendo en cirugías que ya sea dejan la herida abierta o practican cierre primario de ésta, siendo aún discutido cual es la mejor técnica ${ }^{1,2}$. Aunque las técnicas abiertas describen baja recidiva, requieren de tiempo prolongado de cicatrización y curaciones ${ }^{3-5}$. Respecto a las técnicas cerradas, en la última década se ha demostrado que los procedimientos con cierre asimétrico presentan menor recidiva que las de simple cierre primario en la línea media pudiendo estas últimas presentar recidivas de hasta $20 \%{ }^{6,7}$. Dentro de las técnicas asimétricas las más utilizadas son las de colgajo romboidal tipo Limberg o Dufourmentel, apareciendo además en los últimos años trabajos con la técnica de Karydakis que ocupa un colgajo lateral más simple que los anteriores y con baja recidiva ${ }^{8-11}$. Sobre esta técnica hay sólo una experiencia descrita a nivel local ${ }^{12}$. El objetivo de este trabajo es analizar los resultados a corto y largo plazo con la utilización de la técnica de Karydakis en nuestro medio.

\section{Material y Método}

Se analizaron los protocolos de una base de datos prospectiva de pacientes intervenidos por quiste pilonidal con la técnica de Karydakis desde junio de 2005 hasta agosto de 2010. A todos los pacientes que consultaron por quiste pilonidal desde junio de 2005 se les realizó técnica de Karydakis sin haber selección de los pacientes.

Técnica quirúrgica: El procedimiento se inicia luego de la administración de antibioticoprofilaxis al paciente con Cefazolina (1 gr vía endovenosa) y Metronidazol (500 mg vía endovenosa). La técnica utilizada de Karydakis, descrita muy bien por Kitchen ${ }^{13}$, consiste en la realización de una incisión en losanjo lateralizada $2 \mathrm{~cm}$ de la línea media incluyendo el quiste y el tejido que constituye la línea media (Figura 1). Esta resección se realiza hasta la aponeurosis sacra. De preferencia las incisiones se realizan con bisturí frío para evitar la producción de seromas postoperatorios. Luego se talla un colgajo del tejido celular en el borde más medial, de aproximadamente $1 \mathrm{~cm}$ de profundidad y de $2 \mathrm{~cm}$ a lateral. Posteriormente, se cierra el primer plano con puntos de Poliglactina 910 (Vicryl ${ }^{\circledR} 2 / 0$ ) del colgajo inferior de celular a la aponeurosis sacra. En todos los casos se instaló un drenaje tipo redón luego de la realización de la sutura de este plano y posteriormente se cerró el celular en 1 o dos planos. Finalmente, se suturó con puntos de Nylon 3/0 tipo Donatti separados en la piel (Figura 2). En caso de que la fístula se encontrara alejada de la zona del losanjo a resecar, se realizó fistulectomía y cierre del orificio de la piel. El drenaje se retiró a los 2 días y el retiro de puntos se realizó a los 15 días.

Se hizo seguimiento clínico y telefónico de los pacientes. Se consideró falla precoz si presentaba dehiscencia de la herida operatoria después de 15 días. También se definió como recidiva a la supuración de la herida operatoria después de 1 mes desde la cicatrización completa.

Para evaluar la satisfacción de los pacientes, se utilizó una muestra aleatoria de pacientes desde la base de datos prospectiva de este procedimiento. Mediante una encuesta telefónica se abordaron problemas generales de la cirugía, satisfacción del tratamiento con una escala de 1 a 4 , apreciación estética con una escala de 1 a 4 y se les preguntó si recomendarían el procedimiento.

\section{Resultados}

Se analizaron 62 pacientes con un promedio de edad de 24,5 años (15-45), siendo 33 de ellos 


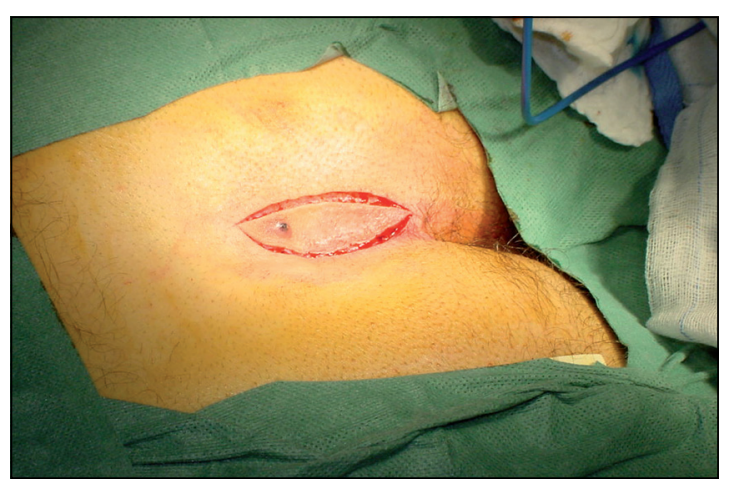

Figura 1. Incisión en losanjo lateralizada hacia la zona más patológica, resecando el surco medio vulnerable.

hombres (53\%). El 20,9\% de los casos presentados eran quistes ya recidivados, la mayoría operados por cierre primario o mediante técnica abierta (Tabla1).

La hospitalización promedio fue de 2,5 días. En el caso de complicaciones precoces, estas ocurrieron en un $14,3 \%$. De ellos el $8 \%$ correspondió a seroma y en un $6,4 \%$ a dehiscencia de sutura. En el primer caso, estas fueron manejadas con punción evacuadoras y en el segundo caso, con curaciones frecuentes llegando a cicatrizar en 1 mes en promedio.

El seguimiento promedio fue de 38 meses (i:12$62)$. Dos pacientes presentaron recidiva (3,2\%). Uno lo presentó a los 6 meses y el otro a los 3 años. Estos fueron resueltos el primero con una nueva cirugía de Karydakis y la segunda con marsupialización.

Para el análisis de satisfacción se entrevistaron a 20 pacientes. De ellos un $80 \%$ no evidenció problemas con el procedimiento. El 20\% restante (4 pacientes) mencionan: cicatriz hipertrófica, dolor prolongado, hipoestesia de la zona y recidiva (descrita previamente). Al pedir a los pacientes que evaluaran su satisfacción con el procedimiento, el $75 \%$ de ellos se declaran muy satisfechos. En cuanto a la estética, el $75 \%$ se declara entre satisfecho y muy satisfecho (Tabla 2). Finalmente, el 95\% de los pacientes recomendaría la realización de este procedimiento en el caso de sufrir esta patología.

\section{Discusión}

La enfermedad pilonidal sacrocoxígea sigue siendo una patología prevalente sin haber hasta el momento claridad de cual es la mejor técnica para resolverla. La discusión sobre cual es su origen se ha ido aclarando hacia el origen adquirido de las lesiones principalmente con los trabajos de Karydakis. Este realizó estudios en 7.471 pacientes de la armada griega concluyendo que son 3 los factores

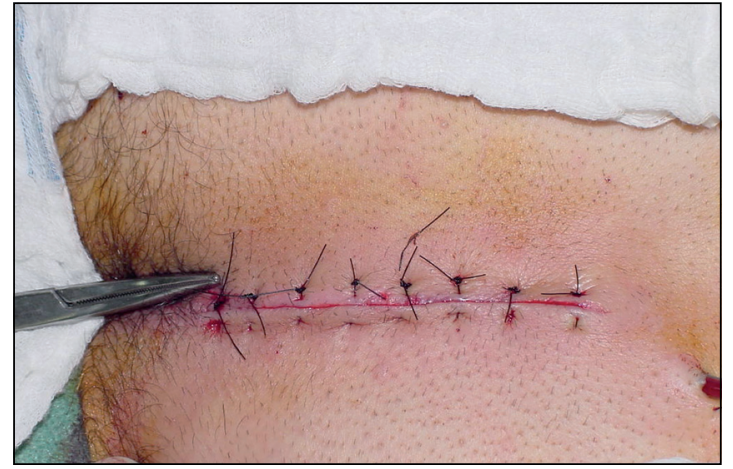

Figura 2. Cierre primario lateralizado señalando la pinza Kelly la línea media, que queda menos hundida.

Tabla 1. Condiciones varias

\begin{tabular}{|lc|}
\hline $\mathrm{n}$ & 62 (hombres 33) \\
Edad & $24,5(15-45)$ \\
Recidivados previos & $20,9 \%$ \\
Hospitalización & 2,5 días \\
Complicaciones precoces & $14,3 \%$ \\
Recidiva & $3,2 \%$ \\
\hline
\end{tabular}

Tabla 2. Satisfacción postoperatoria

\begin{tabular}{|lcc|}
\hline $\begin{array}{l}\text { Grado de } \\
\text { satisfacción }\end{array}$ & $\begin{array}{c}\text { Satisfacción general } \\
\% \text { (n) }\end{array}$ & $\begin{array}{c}\text { Estética } \\
\%(\mathbf{n})\end{array}$ \\
Muy satisfecho & $75 \%(15)$ & $40 \%(8)$ \\
Satisfecho & $20 \%(4)$ & $35 \%(7)$ \\
Poco satisfecho & $5 \%(1)$ & $15(3)$ \\
No satisfecho & $0 \%$ & $10 \%(2)$ \\
\hline
\end{tabular}

que inciden en la ocurrencia de la enfermedad pilonidal sacrocoxígea: la presencia de pelo suelto, las fuerzas de tracción del pelo hacia el surco interglúteo por los movimientos de sentarse y pararse y, finalmente, a la zona vulnerable de la inserción del pelo que es hundida y macerada ${ }^{11}$. Tomando en consideración estos factores, describe su técnica que actúa sobre el factor modificable, que es la zona predispuesta, al sacar los orificios o pits con pelos resecando toda la línea media vulnerable y cerrando la herida paralela a la línea media evitando así una herida en la zona hundida y húmeda que puede predisponer a la dehiscencia y precoz inserción de pelos y a la vez rellenando el surco con este colgajo lateralizado. En su descripción original logra recidiva de un $1 \%$ con una morbilidad de $8,5 \%$ dada princi- 
palmente por infección de la herida ${ }^{11}$. Por otro lado, Bascom también describe la piel hundida del surco natal como predisponente a la inserción de pelo por su maceración y crecimiento bacteriano ${ }^{2}$. La técnica ideal para resolver esta patología debiera ser simple, tener una rápida recuperación y baja recidiva ${ }^{1}$. La técnica abierta más utilizada es la marsupialización con recidivas cercanas al $3 \%$, sin embargo, esta técnica requiere de curaciones de hasta 40 días hasta su cicatrización completa ${ }^{3-5,7}$. Con la técnica de Karydakis el retiro de los puntos se realiza a los 15 días en forma rutinaria, sin requerir más cuidados de la cicatriz en el 94\% de los casos luego de este plazo.

Por otro lado, respecto a las técnicas cerradas, los trabajos de Petersen y una revisión del Cochrane demuestran menor recidiva en las técnicas de cierre asimétrico ${ }^{6,7}$. Dentro de estas están colgajos de v-y, y los más utilizados los de Dufourmentel y Limberg ${ }^{6}$. Esta última ha presentado bajas tasas de recidiva, aunque es técnicamente más demandante. En una reciente serie prospectiva publicada y que compara la técnica de Limberg con la de Karydakis, se demuestra que esta última presenta menores complicaciones (11 vs $20 \%$ ), menor dolor y menor tiempo operatorio, tasas de recidiva similar a 2 años y un mejor resultado estético ${ }^{14}$. Otro estudio comparativo multicéntrico con 72 pacientes por rama logra menor tiempo operatorio en la técnica de Karydakis versus Limberg modificado y una mejor satisfacción de recuperación, sin haber diferencias en las otras variables ${ }^{15}$.

Es interesante que en un trabajo de Ates, se excluyeron pacientes recidivados para el estudio, habiendo nosotros incorporado en nuestra serie estos pacientes (20\% del total de la serie), logrando tanto Ates como nosotros una recidiva de $3 \%$, resultado similar a la mayoría de las series que tienen un seguimiento también prolongado vs $6,9 \%$ para la técnica de Limberg ${ }^{14}$. Esto habla que la técnica de Karydakis es una buena opción incluso en recidivados por otras técnicas.

En una serie se comenta una mayor dificultad de realizar Karydakis si los orificios fistulosos están alejados de la línea media, debiendo en esos casos (7 pacientes) realizar técnica de Limberg. En nuestra experiencia, hemos realizado la fistulectomía con cierre del orificio de piel asociado a la técnica de Karydakis con buen resultado, aunque otras series describen realizar una t con fistulotomía completa y cierre de la piel $^{13}$.

La morbilidad de 14\% coincide con otras experiencias que utilizaron técnica de Karydakis, $(8,5-16 \%)$ siendo las principales complicaciones los seromas y la dehiscencia de sutura de piel manejadas con punción y curaciones (en estas últimas de hasta 1 mes hasta la cicatrización definitiva), sin haber casos de infección de herida en nuestra serie ${ }^{11,15}$. Es fundamental un manejo de rasurado en este período para evitar el anidamiento de pelos en la herida que pueda dar una recidiva precoz.

En nuestra serie utilizamos de rutina el drenaje redón tal como fue sugerida por Karydakis, el que retiramos a las $48 \mathrm{~h}$ habiendo series que discuten si es necesario utilizarlos. Al respecto Gurer demuestra en un trabajo randomizado menor incidencia de seroma con utilización de drenaje aunque la recidiva fue similar ${ }^{16}$.

Sobre la hospitalización de 2,5 días en promedio, esta se explica en el retiro de drenaje en este período, habiendo ya series que manejan los pacientes en forma ambulatoria ${ }^{17,18}$.

Finalmente, la encuesta de satisfacción revela un 95\% de los pacientes que recomendaría esta técnica teniendo una evaluación estética entre satisfactoria y muy satisfactoria en el $75 \%$ de los pacientes. Sería interesante a futuro comparar estos resultados con otras técnicas lo que permitiría dimensionar aun más estos resultados.

En conclusión, la operación de Karydakis logra con una técnica simple y estética una baja recidiva y morbilidad y una recuperación definitiva a los 15 días en la mayoría de los pacientes.

\section{Referencias}

1. Allen-Mersh TG. Pilonidal sinus: finding the right track for treatment. Br J Surg. 1990;77:123-32.

2. Bascom J, Bascom T. Failed Pilonidal Surgery. Arch Surg. 2002; 137:1146-50.

3. Perez G, Bocic G, Azolas C, Garrido R, Jensen C, Valdivia D, y cols. Técnica de marsupialización en quiste pilonidal: 7 años de experiencia. Rev Chil Cir. 1998;50:630-2.

4. Vergara JI, Azolas C, Contador J, Garrido R, Jensen C, Cárdenas R, y cols. Tratamiento quirúrgico del quiste pilonidal. Rev Chil Cir. 1991;43:44-6.

5. Karakayali F, Karagulle E, Karabulut Z, Oksuz E, Moray G, Haberal M. Unroofing and marsupialization vs. rhomboid excision and Limberg flap in pilonidal disease: a prospective, randomized, clinical trial. Dis Colon Rectum 2009;52:496-502.

6. Petersen S, Koch R, Stelzner S, Wendland T, Ludwig K. Primary closure techniques in chronic pilonidal sinus. Dis Colon Rectum 2002;45:1458-67.

7. Al-Khamis, McCallum I, King P, Bruce J. Healing by primary versus secondary intention after surgical treatment for pilonidal sinus. Cochrane Database of Systematic Reviews 2010.

8. Kauer G, Correa R, Rojas H. Utilización del colgajo romboidal simple en el tratamiento quirúrgico de la enfermedad pilonidal. Rev Chil Cir. 1999;51:623-6.

9. Akca T, Colak T, Ustunsoy B, Kanik A, Aydin S. Rando- 
mized clinical trial comparing primary closure with the Limberg flap in the treatment of primary sacrococcygeal pilonidal disease. Br J Surg. 2005;92:1081-4.

10. Karydakis GE. New approach to the problem of pilonidal sinus. Lancet 1973;ii:1414-5.

11. Karydakis GE. Easy and successful treatment of pilonidal sinus after explanation of its causative process. Aust N Z J Surg. 1992;62:385-9.

12. Bannura G, Barrera A, Melo C, Contreras J, Soto D, Mansilla J. Operación de Karydakis en el tratamiento de la enfermedad pilonidal sacrococcígea. Rev Chil Cir. 2005;57:340-4.

13. Kitchen PR. Pilonidal sinus: experience with the Karydakis flap .Br J Surg. 1996;83:1452-5.

14. Ates M, Dirican A, Sarac M, Aslan A, Colak C. Short and long- term results of the Karydakis flap versus the Limberg flap for treating pilonidal sinus disease: a pros- pective randomized study. Am J Surg. 2011;202:568-73.

15. Can M, Sevinc M, Hancerliogullari O, Yilmaz M, Yagci G. Multicenter prospective randomized trial comparing modified Limberg flap transposition and Karydakis flap reconstruction in patients with sacrococcygeal pilonidal disease. Am J Surg. 2010;200:318-27.

16. Gurer A, Gomceli I, Ozdogan M, Ozlem N, Sozen $\mathrm{S}$, Aydin R. Is routine cavity drainage necessary in Karydakis flap operation? A prospective, randomized trial. Dis Colon Rectum 2005;48:1797-9.

17. Anderson J, Yip C, Nagabhushan JS ,Conelly S. Daycase Karydakis flap for pilonidal sinus. Dis Colon Rectum 2008;51:134-8.

18. Bannura G, Barrera A, Contreras J, Melo C, Soto D. Operación de Karydakis ambulatoria en el manejo de la enfermedad pilonidal sacrocoxigea. Rev Chil Cir. 2009;61:256-60. 